\title{
Die Verantwortung des polizeilichen Einsatztrainings
}

\author{
Gesellschaftliche Bilder und Sprachgebrauch in der Bildung von Polizist_innen
}

Die Polizei steht in der Kritik. Zahlreiche Vorfälle, die sich 2020 zugetragen haben oder ans Licht gekommen sind, haben das Vertrauen der Zivilbevölkerung in die Arbeit der Polizei sinken lassen. Dies hat zu einer breiten Diskussion über anzustrebende Veränderungen der polizeilichen Aus-, Fort- und Weiterbildung geführt. Der Text nimmt das polizeiliche Einsatztraining als Aus-, Fort- und Weiterbildungssetting für Polizist_innen in den Blick und dabei den Beitrag, den es möglicherweise zu einer Verbesserung der Situation leisten könnte. Dabei nimmt er von einem reflexiven Standpunkt aus zwei potenzielle blinde Flecken des Trainings in den Blick: Die im Training vermittelten Mensch- und Gesellschaftsbilder und den Sprachgebrauch.

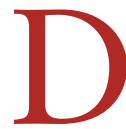
as Bildungspotential des Einsatztrainings und die Vorbildfunktion von Einsatztrainer_innen ist klar dokumentiert (Branch 2020; Staller, Körner, Heil et al., 2021b), wenngleich das Einsatztraining

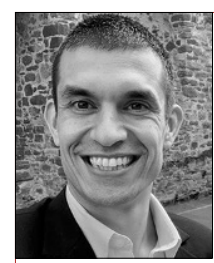

\section{Mario S. Staller}

Hochschule für Polizei und öffentliche Verwaltung NordrheinWestfalen, Aachen, Deutschland

*1982; Prof. Dr. mult., Professor für Psychologie und Training sozialer Kompetenzen an der Hochschule für Polizei und öffentliche Verwaltung Nordrhein-Westfalen, Aachen. mario.staller@hspv.nrw.de

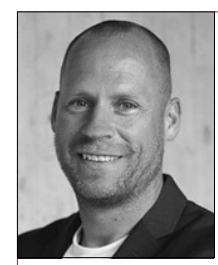

\section{Swen Körner}

Deutsche Sporthochschule Köln, Köln, Deutschland *1975; Univ. Prof. Dr., Professor für Trainingspädagogik und Martial Research an der Deutschen Sporthochschule Köln. koerner@dshs-koeln.de

Zusammenfassung Das polizeiliche Einsatztraining hat eine große Bedeutung: Junge Polizist_innen werden dort im Rahmen ihrer Erstsozialisation ausgebildet, in der Praxis tätige Polizist_innen in einsatz- und konfliktrelevanten Bereichen geschult und weiterentwickelt. Neben diesen Inhalten werden innerhalb des Lehr-Lern-Settings explizit und implizit auch Werte, Einstellungen und Sichtweisen auf polizeiliche Einsatz- und Konfliktsituationen vermittelt. Der Beitrag zeigt die Verantwortung des polizeilichen Einsatztrainings auf, zu einer sozialen und gerechten Gesellschaft beizutragen. Am Beispiel (a) der vermittelten Gesellschaftsbilder und (b) der verwendeten Sprache skizzieren die Autoren für das polizeiliche Einsatztraining Anlässe und Potenziale, sich dieser Verantwortung reflektiert zu stellen.

Schlüsselwörter Polizei, Einsatztraining, Gesellschaftsbilder, Sozialisation als Forschungsgegenstand bisher - gerade in Deutschland - eher weniger als Sozialisationsinstanz wahrgenommen wurde und erst jüngst auch in den Fokus polizeiwissenschaftlicher Forschung geraten ist. Während im Einsatztraining regelmäßig Fragestellungen der Trainingsoptimierung im Mittelpunkt stehen, ist von einer reflektierten professionellen Praxis zudem zu erwarten, dass sie regelmäßig zu sich selbst in Distanz tritt und das eigene Handeln von außen betrachtet. Es geht dann nicht ausschließlich um die Frage, was man tut, sondern darum, wie man tut, was man tut - und warum. Ein derartiger reflexiver Mechanismus (Koerner 2015) eröffnet den Blick auf Voraussetzungen, Folgen und Latenzen des eigenen Tuns. Dabei können u. a. (un-)beabsichtigt mitschwingende Werte oder Einstellungen kontrolliert werden, die möglicherweise im Rahmen des Einsatztrainings Wirkung entfalten (Staller et al. 2019). Wir legen im Folgenden einige Aspekte dar, die uns im Rahmen einer reflexiven Gestaltung des Einsatztrainings und zur Gestaltung einer modernen, bürgerorientieren Polizei wichtig erscheinen und für die es Anhaltspunkte gibt, dass gerade sie blinde Flecken innerhalb des Einsatztrainings bilden. Ihre Sichtbarmachung helfen kann, die „control complexity “ (Luhmann 2008, S. 157) im System der Polizei zu erhöhen.

\section{Verantwortungsbereich 1: Bilder der Gesellschaft und der Polizei}

Das Einsatztraining transportiert Bilder der Gesellschaft und der Polizei, welche ihrerseits im Verhalten von Polizeibeamt_innen Wirkung entfalten (Branch 2020). Dies geschieht z. B. über die vermittelten Inhalte, über das Verhalten des/r Trainer_in oder über die eingesetzte Literatur. Sich dieser Bilder bewusst zu sein 
und deren Inhalt und Wirkung kritisch zu reflektieren, ist von essentieller Bedeutung für Beziehung zwischen Polizei und Zivilgesellschaft.

\section{Gefährlichkeit des Polizeiberufs}

Ein rekurrierend transportiertes Bild ist dabei das der Gefährlichkeit des Polizeiberufs. Das Narrativ, dass der Polizeiberuf sehr gefährlich sei, hält sich innerhalb der Polizei und wird dort kontinuierlich weitergegeben und als Fakt bewertet (Branch 2020; Gibbs 2019; Kurtz und Upton 2017a; Lynch 2017; Marenin 2016). Neben verschiedenen Akteuren (Polizeigewerkschaften, Politiker_innen, Entscheider_innen, Medien etc.) wird das Narrativ auch von Einsatztrainer_innen regelmäßig weiterverbreitet (Branch 2020): Entweder direkt („Don't let them kill you on some dirty roadway“ Lynch 2017) oder indirekt durch das überproportionale Trainieren von hochgradig gefährlichen Situationen, mit welchem der Bedarf für das Einsatztraining bei den Lerner_innen geschaffen werden soll (Staller et al. 2019).

Auch Verbände und Trainingsorganisation, wie beispielsweise „Polizeitrainer in Deutschland e. V.“, haben an der Erhaltung und dem weiteren Ausbau dieser narrativen Konstruktion einen Anteil. So zeigen Coverbilder des jährlich erscheinenden Magazins des Vereins seit seiner Gründung 1996 überwiegend Darstellungen, die explizit auf die Gefährlichkeit des Polizeiberufs hinweisen sollen. Auch bei den einmal jährlich stattfindenden Polizeitrainerfachkonferenzen, an denen regelmäßig mehrere hundert Einsatztrainer_innen aus dem deutschsprachigen Raum teilnehmen, finden sich derartige Bezüge in Form von Beiträgen (Polizeitrainer in Deutschland 2019). Thematisch nimmt die Gefährlichkeit des Polizeiberufs einen prominenten Raum ein; die polizeiliche Zwangsanwendung steht im Mittelpunkt (Polizeitrainer in Deutschland, 2019). Untersuchungen zur Wissensgenerierung von Einsatztrainer_innen zeigen, dass gerade der Austausch auf derartigen Symposien mit Kollegen_innen einen besonderen Wert hat (Staller et al. 2021a). Die hier verhandelten Narrative spielen für die Wissenskonstruktion von Einsatztrainer_innen über Mensch und Gesellschaft eine nicht zu unterschätzende Rolle.

Auch ein Blick auf die populärwissenschaftliche Literatur zur Einsatzbewältigung zeigt, dass auch hier das Bild von der Gefährlichkeit des Polizeiberufs regelmäßig bedient wird (Asken und Grossman 2010; Grossman und Christensen 2007). Ein nationales Beispiel (Füllgrabe 2014; Grandel 2015; Schmidt 2017) liest sich wie folgt: Der polizeiliche „Krieger“ stellt sich der Gefahr, die „[...] ein ständiger Begleiter des Polizeibeamten, jeden Tag [...]“ ist (Grandel 2015, S. 59).
Die hier zu vernehmende Kriegermentalität ist für eine demokratisch orientierte Polizeiarbeit problematisch (Körner und Staller 2020; Stoughton 2015). Demokratische Formen der Polizeiarbeit konzentrieren sich auf die Schaffung von Legitimität durch Verfahrensgerechtigkeit, die in einer fairen und respektvollen Behandlung wurzelt, sowie auf gemeinschaftsorientierte Polizeiprogramme, die auf die Gesellschaft als Ganzes ausgerichtet und partizipativ sind (Trinkner et al. 2016). Die Kriegermentalität hingegen fördert ein Selbstbild von Polizist_ innen als Soldaten an der Front in einem nicht enden wollenden Kampf zur Bewahrung von Ordnung und Zivilisation gegen die Kräfte des Chaos und der Kriminalität. Der Aufbau von Vertrauen zwischen Polizei und Bürger_innen fällt hier hinter Herangehensweisen zurück, welche als misstrauisch und auf Konfrontation angelegt beschrieben werden können (Stoughton 2016). Das Narrativ der ständigen Gefahr verstärkt diese Kriegermentalität (Branch 2020; Stoughton 2015).

Dabei sprechen die Zahlen eine andere Sprache: Im historischen Längsschnitt sowie im Vergleich zu anderen Berufen ist der Polizeiberuf sicherer denn je (Gibbs 2019; Staller und Körner 2019). Im Gegensatz dazu normalisiert sich durch kommunikative Wiederholung die Vorstellung einer allgegenwärtigen Gefahr der Polizeiarbeit, die auf empirisch fragwürdigen Annahmen basiert. Die Schlussfolgerung ist auch in Hinsicht auf ihre Folgen problematisch: Forschungen deuten darauf hin, dass mit einer subjektiv erhöhten wahrgenommen Gefährlichkeit des Berufs auch ein höheres Gewalterleben im Dienst einhergeht (Baier 2020) und subjektiv wahrgenommene Antipathie der Gesellschaft gegenüber der eigenen polizeilichen Berufsgruppe zu mehr Zwang und einer Abkapselung von der Zivilgesellschaft (Boivin et al. 2018; Marier und Moule 2019) führt. Die Wahrscheinlichkeit zur Ausübung von Polizeigewalt steigt dadurch (Marenin 2016). Aus aggressionspsychologischer Sicht ergeben sich weitere Problematiken: Uneindeutige Verhaltensweisen von Bürger_innen werden als Anzeichen einer bevorstehenden Gefahr wahrgenommen (Kahn et al. 2017). Die Wahrscheinlichkeit zur Ausübung von aggressiven Handlungen steigt dadurch, da sich Polizist_innen durch das missinterpretierte Verhalten bedroht fühlen. Wenn die eigene subjektive Welt als gefährlicher Ort wahrgenommen wird, erhöht sich die Wahrscheinlichkeit, selbst aggressive Handlungen vorzunehmen (Huesmann 2018). Der Transport eines empirisch fragwürdigen Narrativs ist demnach mit beachtenswerten Kosten verbunden: eine Steigerung aggressiven Verhaltens durch Polizist_innen. 


\section{Stereotype}

Ein Blick auf die innerhalb der polizeilichen Aus- und Fortbildung verwendete Literatur zeigt, dass hier teilweise Stereotype transportiert werden, welche eines zweiten Blicks bedürfen und ebenfalls auf ihre Voraussetzungen und Folgen hin zu prüfen sind. Dies betrifft u. a. stereotype Bilder zur (angenommenen) Herkunft von Menschen (z. B. Heubrock et al. 2012), verwandtschaftliche Verbindungen zu Familien (z. B. Dienstbühl 2019) oder zum mentalen Gesundheitszustand von Menschen (Staller, unveröffentlichte Daten aus Hausarbeiten). Die negativen Auswirkungen stereotypischer und stigmatisierender Bilder auf das gesamtgesellschaftliche Zusammenleben sowie gleichberechtigter Teilhabe (Boettner und Schweitzer 2020) zeigen sich auch in der PolizeiBürger_innen-Interaktionen in Form erhöhter stereotypbasierter Kontrollen (Kahn und Martin 2020) sowie einem Mehr an Zwangshandeln (Kahn et al. 2017). Auch antiquierte Gender- und Altersstereotype werden innerhalb der Polizei berichtet (Hunold 2019) und innerhalb des Einsatztrainings (Cushion 2020; Staller et al. 2019) transportiert: die Frau als nicht geeignetes Individuum zur Konfliktlösung im Einsatz, die neue Generation als körperlich dahinsiechende Kohorte. Empirische Befunde weisen auf andere Realitäten hin (Jaeckle et al. 2019).

\section{Diversität}

Die Polizei tut sich mit Diversität noch schwer (Genkova 2019). Doch was im Großen für die Polizeiorganisation gilt, erscheint auch für das Einsatztraining beachtenswert. Daten aus Erhebungen zum biographischen Hintergrund von Einsatztrainer_innen (Körner et al. 2019) legen das Bild eines prototypischen Einsatztrainers nahe: weiß, männlich, ohne Migrationshintergrund, erfahren in der Anwendung physischer Mittel durch Kampfsport, Verwendung in polizeilichen Spezialeinheiten oder geübt im Umgang mit Schusswaffen. Während diese Eigenschaften im Einzelfall nicht problematisch sind und zudem in Bezug auf die Konfliktlösekompetenz wichtige fachliche Expertisen ausweisen, geht auf einer strukturellen Ebene damit die Gefahr eines verzerrten Welt- und Berufsbildes einher. Wer etwa vor allem zu kämpfen gelernt hat, muss seine sozialisatorisch eingeschriebenen Handlungspräferenzen in Bezug auf andere Sichtweisen und Perspektiven der polizeilichen Einsatzbewältigung überprüfen. Geschieht dies nicht und wird dies auch nicht auf behördlicher Ebene etwa durch Rekrutierungskriterien und Fortbildungen eingefordert, steigt die Wahrscheinlichkeit des Transportes von homogenen Welt-, Gesellschafts- und Konfliktbewältigungsbildern.

\section{Storytelling}

Der Transport von Narrativen und stereotypen Weltund Gesellschaftsbildern erfolgt auf unterschiedlichsten Wegen: durch Literatur, durch die Auswahl von Trainingsinhalten, durch den Umgang mit Lerner_innen. Ein besonders hervorstechender - weil auch gut erforschter Aspekt - ist das Storytelling, das Geschichtenerzählen innerhalb des Polizeisystems. Das Erzählen von Geschichten trägt maßgeblich dazu bei, das Bild der Gesellschaft, das Wesen der Polizeiarbeit von Polizist_innen sowie Stereotype, Hierarchien und Dominanzstrukturen zu bilden, zu festigen und zu verstärken (Branch 2020; van Hulst 2013, 2017; Kurtz und Upton 2017b; Woods, 2019). Polizeiliche Lehrkräfte nutzen das Geschichtenerzählen in besonderem Maße (Branch 2020; Lynch 2017). Welche Geschichten wie erzählt und welche Bilder dabei reproduziert werden, liegt ebenfalls eine Verantwortung eines aufgeklärten, sich seiner eigenen Annahmen bewussten Einsatztrainings. Wiederkehrende, verzerrende und vor allem international bereits erforschte Themen beinhalten $u$. a.

- die Gefährlichkeit des polizeilichen Tätigwerdens (Branch 2020; Lynch 2017) - trotz anderslautender empirischer Fakten zur (Gibbs 2019);

- die Notwendigkeit einer militaristischen Einstellung, um mit der narrativ transportierten Annahme der täglichen Gefährdung umzugehen (Boe et al. 2020; Kurtz und Upton 2017a; Lynch 2017) - obwohl andere Einstellungen ethisch-normativ gebotener und zielführender erscheinen (Körner und Staller 2020; Stoughton 2015);

- Genderstereotype, die männliche Dominanz innerhalb des Polizeiberufs verfestigen, indem männliche Polizisten als Helden und weibliche Polizistinnen und jüngere Kolleg_innen als hilfebedürftige Opfer (gerade in Konfliktsituationen) konstruiert werden (Kurtz und Upton 2017b) - obwohl Studien zur Konfliktlösekompetenz hier anderes nahe legen (Jaeckle et al. 2019);

- den Polizeiberuf als kontinuierlich spannender, herausfordernder und aufregender Beruf, in dem viel passiert (Branch 2020) - obwohl auch häufig mal nichts zu tun ist und Langweile dominiert (Phillips 2016).

Die genannten Aspekte geben Anlass zur Reflexion: Welche Weltbilder werden transportiert? Welche Stereotypen reproduziert? Welche Geschichten werden erzählt? Welche nicht? Eine kritische Reflexion darauf bildet die Grundvoraussetzung professionellen Handeln im System der Polizei. Professionelle Supervision wie sie in Tätigkeitsfeldern der sozialen Arbeit üblich ist (Sewell 2018), könnte hier helfen, gemachte Erfahrungen einzuordnen, die damit verbundenen Affekte und Emotionen 
sowie deren biografische Einflüsse zu reflektieren und eine mehrdimensionale Bewertung und Gestaltung erlebter Situationen vorzunehmen (Rieforth und BeermannKassner 2017). Auch könnten Hospitationen von Polizist_innen in Handlungsfeldern der sozialen Arbeit dazu beitragen, Perspektiven und Sichtweisen auf Lebensentwürfe, Biografien und soziale Problemstellungen anderer Menschen gewinnbringend zu erweitern (Behr 2018).

\section{Verantwortungsbereich 2: Sprache}

Die Sprache prägt unser subjektives Verständnis der Realität (Felder 2009) und beeinflusst das Handeln (Black et al. 1981). Damit kommt ihrer bewussten Nutzung gerade in Bildungskontexten wie dem Einsatztraining eine enorme Bedeutung zu. Was im Einsatztraining gesagt wird, was in Handreichungen und in entsprechender Literatur wie geschrieben steht, hat Einfluss auf die Sichtweise der Rezipient_innen. Entsprechend hat das Einsatztraining auch hier eine Verantwortung zum reflexiven Umgang. Dies ist gerade vor dem Hintergrund von Fällen wichtig, in denen über Sprache Abwertungen gegenüber bestimmten Menschengruppen geäußert wird (Radburn et al. 2020; Voigt et al. 2017). Das Einsatztraining hat hier gerade in seiner sozialisierenden Wirkung Vorbildfunktion: Das Nutzen einer konsequent vorurteilsfreien und inklusiven Sprache. Dies betrifft

- die Repräsentation aller Geschlechter (z. B. „Liebe Damen, Herren und Diverse“, „Hey zusammen“, etc.);

- das Nutzen einer personenzentrierten Sprache (z. B. „Menschen ohne Obdach“);

- das Nutzen einer entstigmatisierenden Sprache (z. B. „Mensch in der Krise“, „Mensch mit einem Alkoholproblem“ etc.);

- die Präzisierung von Menschen, durch tätigkeitsorientierte Zuschreibungen (z. B. „ein Mensch, der gerade nackt in den Brunnen steigt") und

- Vermeidung von gruppenorientierten Zuschreibungen (z. B. in Bezug auf Geschlecht, Herkunft, Familienzugehörigkeit, mentaler Gesundheitszustand, Drogenproblematik, etc.).

Studienergebnisse legen nahe, dass im Laufe der polizeilichen Sozialisation vermehrt eine Abkapselung von der Zivilgesellschaft erfolgt und eine „Wir-gegen-die“Mentalität entsteht (Boivin et al. 2018). Der Fokus auf die Generierung und Erhaltung einer sozialen Identität als Polizei auf der einen Seite (wir) im Vergleich zu den „Anderen“ auf der anderen Seite (die) scheint hierbei besonders problematisch, da hierdurch zwei soziale Identitäten verfestigt werden, die eigentlich zu einer gehören: der Gesellschaft (Radburn et al. 2020). Narra- tive wie beispielsweise das der Gefährlichkeit oder der Einzigartigkeit des Polizeiberufs tragen ihren Teil dazu bei, die getrennten sozialen Identitäten zu manifestieren (Branch 2020). Gerade mit Blick auf gerechtes polizeiliches Verhalten zeigen Konzepte wie die Theorie der prozeduralen Gerechtigkeit, dass sich dadurch Polizei-Bürger_innen-Interaktionen verbessern lassen (Antrobus et al. 2019; Wood et al. 2020). Die Grundlage hierfür ist ein gemeinsames Verständnis als eine soziale Gruppe: unsere Gesellschaft (Radburn et al. 2020).

Vor diesem Hintergrund müssen auch polizeilich genutzte Begrifflichkeiten in Frage gestellt werden. Das begrifflich häufig genutzte „polizeiliche Gegenüber“ impliziert z. B. eine Gegenposition: Der Begriff prägt das Konzept des Gegners. Und Gegner werden „dominiert“. Ein Verb, das sich auch so in polizeilichen Handlungsanweisungen wiederfindet (Dienstbühl 2019, S. 14). Entsprechend macht es einen Unterschied im Denken und Handeln, ob Adressat_innen einer polizeilichen Maßnahme als Bürger_innen oder als Gegenüber gesehen werden (Zaiser und Staller 2015). Das eine (Bürger_innen) stellt eine gemeinsame soziale Identität in den Mittelpunkt, schließlich ist der oder die Polizist_in selbst Bürger_in, und ermöglicht damit eher Zugang zu kooperativeren Konfliktlösungen, das andere (Gegenüber) stellt die Differenz, und damit eine andere soziale Identität als die eigene in den Mittelpunkt. Kooperative Konfliktlösung wird dadurch erschwert (Zaiser und Staller 2015), die Abgrenzung der Polizei wahrscheinlicher (Boivin et al. 2018). Entsprechend erachten wir es als geboten, Interaktionspartner_innen polizeilichen Handelns als Bürger_in oder eben ganz kommunikationstheoretisch, als Adressat_in einer Maßnabme oder Empfänger_in, zu benennen.

\section{Fazit}

Einsatztrainer_innen sind Vorbilder, für Bürger_innen allgemein und für junge Polizist_innen im Besonderen (Staller, Körner, Heil et al., 2021b); Einsatztraining hat einen großen Einfluss auf die Sozialisation von Polizist_innen (Branch 2020). Dem Einsatztraining kommt damit eine wichtige Verantwortung hinsichtlich Veränderungen innerhalb der Polizei zu. Wir haben zwei Verantwortungsbereiche skizziert, deren reflexiver Prüfung und Gestaltung sich das Einsatztraining stellen muss: die vermittelnden Gesellschaftsbilder und der Sprachgebrauch. Mit Blick auf die Entwicklung polizeilicher Reflexivität bieten sich zum Beispiel Hospitationen in Berufen der Sozialen Arbeit an (Behr 2018). Die temporäre Teilhabe in diversen Handlungsfeldern der Sozialen Arbeit (Kinder- und Jugendsozialarbeit etc.) sowie Supervision könnten für Einsatztrainer_innen und Poli- 
zist_innen wertvolle Erfahrungen, Perspektivenerweiterungen und Reflexionsmöglichkeiten bereithalten.

Eingegangen. 16. Dezember 2020

Angenommen. 26. Januar 2021

Funding. Open Access funding enabled and organized by Projekt DEAL.

Open Access. Dieser Artikel wird unter der Creative Commons Namensnennung 4.0 International Lizenz veröffentlicht, welche die Nutzung, Vervielfältigung, Bearbeitung, Verbreitung und Wiedergabe in jeglichem Medium und Format erlaubt, sofern Sie den/die ursprünglichen Autor(en) und die Quelle ordnungsgemäß nennen, einen Link zur Creative Commons Lizenz beifügen und angeben, $o b$ Änderungen vorgenommen wurden.

Die in diesem Artikel enthaltenen Bilder und sonstiges Drittmaterial unterliegen ebenfalls der genannten Creative Commons Lizenz, sofern sich aus der Abbildungslegende nichts anderes ergibt. Sofern das betreffende Material nicht unter der genannten Creative Commons Lizenz steht und die betreffende Handlung nicht nach gesetzlichen Vorschriften erlaubt ist, ist für die oben aufgeführten Weiterverwendungen des Materials die Einwilligung des jeweiligen Rechteinhabers einzuholen.

Weitere Details zur Lizenz entnehmen Sie bitte der Lizenzinformation auf http://creativecommons.org/licenses/by/4.0/deed.de.

\section{Literatur}

Antrobus, E., Thompson, I., \& Ariel, B. (2019). Procedural justice training for police recruits: results of a randomized controlled trial. Journal of Experimental Criminology, 15(1), 29-53. https://doi.org/10.1007/s11292018-9331-9.

Asken, M. J., \& Grossman, D. (2010). Warrior mindset. Millstadt: Warrior Science Publications.

Baier, D. (2020). Antworten auf Huhn-und-Ei-Fragen: Was Längsschnittstudien leisten können, illustriert am Beispiel des Zusammenhangs von Gewalterfahrungen und Furcht vor Übergriffen. Format Magazine, 9, 25-31. Answers to chicken and egg questions: What longitudinal studies can achieve illustrated by the example of the connection between experiences of violence and fear of assault.

Behr, R. (2018). Rassismus und Diskriminierung im Polizeidienst - Die Karriere zweier „Reizworte“. SIAK Journal, 2, 57-66. https:// doi.org/10.7396/2018_2_f.

Black, M., Lakoff, G., \& Johnson, M. (1981). Metaphors we live by. The Journal of Aesthetics and Art Criticism, 40(2), 208. https:// doi.org/10.2307/430414.

Boe, O., Torgersen, G.-E., \& Skoglund, T. H. (2020). Does the Norwegian police force need a well-functioning combat mindset? Frontiers in Psychology. https://doi.org/10.3389/fpsyg.2020.01075.

Boettner, J., \& Schweitzer, H. (2020). Der Name als Stigma. Sozial Extra. https://doi.org/10.1007/s12054-020-00330-2.

Boivin, R., Faubert, C., Gendron, A., \& Poulin, B. (2018). The "us vs them" mentality: a comparison of police cadets at different stages of their training. Police Practice and Research, 5(1), 1-13. https://doi.org/10.1080/1 5614263.2018.1555480.

Branch, M. (2020). "The nature of the beast:" the precariousness of police work. Policing and Society. https://doi.org/10.1080/10439463.2020.18 18747.

Cushion, C. J. (2020). Exploring the delivery of officer safety training: a case study. Policing: A Journal of Policy and Practice, 14(1), 166-180. https://doi.org/10.1093/police/pax095.
Dienstbühl, D. (2019). Arabische Famlienclans - Historie. Analyse. Ansätze zur Bekämpfung. https://www.presseportal.de/blaulicht/ pm/11562/4717559 Polizeipräsidium Essen. Zugegriffen: 20. Jan. 2021.

Felder, E. (2009). Sprache - das Tor zur Welt!? In E. Felder (Hrsg.), Sprache (S. 13-57). Berlin, Heidelberg: Springer. https://doi.org/10.1007/978-3642-00342-4_2.

Füllgrabe, U. (2014). Psychologie der Eigensicherung. Boorberg.

Genkova, P. (2019). Diversity und Polizei. In H. J. Lange, T. Model \& M. Wendekamm (Hrsg.), Zukunft der Polizei (S. 113-131). Wiesbaden: Springer VS. https://doi.org/10.1007/978-3-658-22591-9_8.

Gibbs, J. C. (2019). Rhetoric vs. reality of decreasing danger for police over time. Criminology \& Public Policy, 18(1), 7-10. https:// doi.org/10.1111/1745-9133.12423.

Grandel, U. (2015). Selbstverteidigung in der Polizei. Boorberg.

Grossman, D., \& Christensen, L. W. (2007). On combat: the psychology and physiology of deadly conflict in war and peace. Millstadt: Warrior Science Publications.

Heubrock, D., Boberg, W., Kolewe, B., Lübben, J., \& Orböck, S. (2012). Türken und Araber verstehen und vernehmen: Empfehlungen zur interkulturellen Vernehmung arabisch-türkischer Personen. Frankfurt, M.: Verlag für Polizeiwissenschaft.

Huesmann, L. R. (2018). The contagion of violence. In A. T. Vazsonyi, D. J. Flannery \& M. DeLisi (Hrsg.), The Cambridge handbook of violent behavior and aggression (S. 527-556). Cambridge: Cambridge University Press.

van Hulst, M. (2013). Storytelling at the police station: the canteen culture revisited. British Journal of Criminology, 53(4), 624-642. https:// doi.org/10.1093/bjc/azt014.

van Hulst, M. (2017). Backstage storytelling and leadership. Policing: A Journal of Policy and Practice, 11(3), 356-368. https://doi.org/10.1093/police/pax027.

Hunold, D. (2019). „Wer hat jetzt die größeren Eier?!“ - Polizeialltag, hegemoniale Männlichkeit und reflexive Ethnografie. In C. Howe \& L. Ostermeier (Hrsg.), Polizei und Gesellschaft - Transdisziplinäre Perspektiven zu Methoden, Theorie und Empirie reflexiver Polizeiforschung (S. 4769). Wiesbaden: Springer. https://doi.org/10.1007/978-3-658-22382-3_3. "Who's got the bigger balls now?! “ - Everyday police life, hegemonic masculinity and reflective ethnography.

Jaeckle, T., Benoliel, B., \& Nickel, O. (2019). Police use of force decisions: a gender perspective. In Walden University Research Symposium - 2019 Program \& Posters Bd. 23. Tampa: Walden University.

Kahn, K. B., \& Martin, K. D. (2020). The social psychology of racially biased policing: evidence-based policy responses. Policy Insights from the Behavioral and Brain Sciences, 7(2), 107-114. https:// doi.org/10.1177/2372732220943639.

Kahn, K. B., McMahon, J. M., \& Stewart, G. (2017). Misinterpreting danger? Stereotype threat, pre-attack indicators, and police-citizen interactions. Journal of Police and Criminal Psychology, 20(1), 316. https:// doi.org/10.1007/s11896-017-9233-1.

Koerner, S. (2015). Reflexive Mechanismen und Sportwissenschaft. In S. Koerner \& V. Schürmann (Hrsg.), Reflexive Sportwissenschaft. Konzepte und Fallanalysen. Reflexive Sportwissenschaft, (Bd. 1, S. 129-143). Berlin: Lehmanns.

Körner, S., \& Staller, M. S. (2020). Commentary: does the Norwegian police force need a well-functioning combat mindset? Frontiers in Psychology. https://doi.org/10.3389/fpsyg.2020.572324.

Körner, S., Staller, M. S., \& Kecke, A. (2019an). „Weil mein Background da war ..." - Biographische Effekte bei Einsatztrainern"innen. In M. Meyer \& M. S. Staller (Hrsg.), Lehren ist Lernen: Methoden, Inhalte und Rollenmodelle in der Didaktik des Kämpfens": internationales Symposium; 8. Jahrestagungder dvs Kommission „Kampfkunst und Kampfsport" vom 3.-5. Oktober 2019 an der Universität Vechta; Abstractband (S. 17-18). Hamburg: Deutsche Vereinigung für Sportwissenschaften (dvs). "Because my background was ... - Biographical effects for police trainers".

Kurtz, D. L., \& Upton, L. (2017a). War stories and occupying soldiers: a narrative approach to understanding police culture and community conflict. Critical Criminology, 25(4), 539-558. https://doi.org/10.1007/s10612017-9369-4.

Kurtz, D. L., \& Upton, L. L. (2017b). The gender in stories: how war stories and police narratives shape masculine police culture. Women \& Criminal Justice, 28(4), 1-19. https://doi.org/10.1080/08974454.2017.1294132. 
Luhmann, N. (2008). Die Ausdifferenzierung des Erkenntnisgewinn: Zur Genese von Wissenschaft. In A. Kieserling (Hrsg.), Ideenevolution: Beiträge zur Wissenssoziologie (S. 132-185). Frankfurt, M.: Suhrkamp.

Lynch, C. G. (2017). Don't let them kill you on some dirty roadway: survival, entitled violence, and the culture of modern American policing. Contemporary Justice Review, 21(1), 1-11. https://doi.org/10.1080/10282580. 2018.1415045.

Marenin, O. (2016). Cheapening death: danger, police street culture, and the use of deadly force. Police Quarterly, 19(4), 461-487. https:// doi.org/10.1177/1098611116652983.

Marier, C. J., \& Moule, R. K. (2019). Feeling blue: officer perceptions of public antipathy predict police occupational norms. American Journal of Criminal Justice, 44(5), 836-857. https://doi.org/10.1007/s12103-018 9459-1.

Phillips, S. W. (2016). Police discretion and boredom. Journal of Contemporary Ethnography, 45(5), 580-601. https:// doi.org/10.1177/0891241615587385.

Polizeitrainer in Deutschland. (2019). EPTK 2019-Themen und Referenten am Mittwoch dem 06.03.2019. https://www.polizeitrainer.de/news/konferenzen/eptk-2019/. Zugegriffen: 20. Jan. 2021

Radburn, M., Savigar-Shaw, L., Stott, C., Tallent, D., \& Kyprianides, A. (2020). How do police officers talk about their encounters with "the public"? Group interaction, procedural justice and officer constructions of policing identities. Criminology \& Criminal Justice. https:// doi.org/10.1177/1748895820933912.

Rieforth, J., \& Beermann-Kassner, A. (2017). Selbsterfahrung und Supervision. Konfliktdynamik, 6(2), 104-113. https://doi.org/10.21706/kd-6-2104.

Schmidt, R. (2017). Das Phänomen Zweikampf. S. Ka.-Verlag.

Sewell, K. M. (2018). Social work supervision of staff: a primer and scoping review (2013-2017). Clinical Social Work Journal, 46(4), 252-265. https://doi.org/10.1007/s10615-018-0679-0.

Staller, M. S., \& Körner, S. (2019). Quo vadis Einsatztraining? In E. Kühne (Hrsg.), Die Zukunft der Polizeiarbeit - die Polizeiarbeit der ZukunftTeil II (S. 321-364). Rothenburg: Eigenverlag der Hochschule der Sächsischen Polizei (FH). Quo vadis police training?

Staller, M. S., Körner, S., Abraham, A., \& Poolton, J. (2021a). Topics, Sources and the Application of Coaching Knowledge in Police Training. Under Review.

Staller, M. S., Körner, S., Heil, V., \& Kecke, A. (2019). Mehr gelernt als geplant? Versteckte Lehrpläne im Einsatztraining [More learned than planed? The hidden curriculum in police use of force training. In B. Frevel \& P. Schmidt (Hrsg.), Empirische Polizeiforschung XXII Demokratie und Menschenrechte - Herausforderungen für und an die polizeiliche Bildungsarbeit (S. 132-149). Frankfurt: Verlag für Polizeiwissenschaft.

Staller, M. S., Körner, S., Heil, V., Abraham, A., \& Poolton, J. (2021b). Police recruits' wants and subjective needs in mandatory police training. Under Review.

Stoughton, S. W. (2015). Law enforcement's “warrior” problem. Harvard Law Review Forum, 128(225), 225-234.

Stoughton, S. W. (2016). Principled policing: warrior cops and guardian officers. Wake Forest Law Review, 51, 611-667.

Trinkner, R., Tyler, T. R., \& Goff, P. A. (2016). Justice from within: the relations between a procedurally just organizational climate and police organizational efficiency, endorsement of democratic policing, and officer well-being. Psychology, Public Policy, and Law, 22(2), 158-172. https:// doi.org/10.1037/law0000085.

Voigt, R., Camp, N. P., Prabhakaran, V., Hamilton, W. L., Hetey, R. C., Griffiths, C. M., Jurgens, D., Jurafsky, D., \& Eberhardt, J. L. (2017). Language from police body camera footage shows racial disparities in officer respect. Proceedings of the National Academy of Sciences, 114(25), 65216526. https://doi.org/10.1073/pnas.1702413114.

Wood, G., Tyler, T. R., \& Papachristos, A. V. (2020). Procedural justice training reduces police use of force and complaints against officers. Proceedings of the National Academy of Sciences of the United States of America, 117(18), 9815-9821. https://doi.org/10.1073/pnas.1920671117.

Woods, J. B. (2019). Policing, danger narratives, and routine traffic stops. Michigan Law Review, 117(4), 635-712.

Zaiser, B., \& Staller, M. S. (2015). The word is sometimes mightier than the sword: rethinking communication skills to enhance officer safety. Journal of Law Enforcement, 4(5), 1-17.

Hier steht eine Anzeige.

Springer 